\title{
Experimental Exploration of RSSI Model for the Vehicle Intelligent Position System
}

\author{
Zhichao Cao*, Zhenzhou Yuan, Silin Zhang \\ MOE Key Laboratory for Urban Transportation Complex Systems Theory and Technology, \\ Beijing Jiaotong University (China) \\ *Corresponding authorcba0.10.18@163.com, zavuan@bjtu.edu.cn, 12114213@bjitu.edu.cn
}

Received: September 2014

Accepted: January 2015

\section{Abstract:}

Purpose: Vehicle intelligent position systems based on Received Signal Strength Indicator (RSSI) in Wireless Sensor Networks (WSNs) are efficiently utilized. The vehicle's position accuracy is of great importance for transportation behaviors, such as dynamic vehicle routing problems and multiple pedestrian routing choice behaviors and so on. Therefore, a precise position and available optimization is necessary for total parameters of conventional RSSI model.

Design/methodology/approach: In this paper, we investigate the experimental performance of translating the power measurements to the corresponding distance between each pair of nodes. The priori knowledge about the environment interference could impact the accuracy of vehicles' position and the reliability of parameters greatly. Based on the real-world outdoor experiments, we compare different regression analysis of the RSSI model, in order to establish a calibration scheme on RSSI model.

Findings: Empirical experimentation shows that the average errors of RSSI model are able to decrease throughout the rules of environmental factor $\mathrm{n}$ and shadowing factor $\eta$ respectively. Moreover, the calculation complexity is reduced, as an innovative approach. Since variation tendency of environmental factor $\mathrm{n}$, shadowing factor $\eta$ with distance and signal strength could be simulated respectively, RSSI model fulfills the precision of the vehicle intelligent position system. 
Research limitations/implications: In this research, it is not evident to find the variation trend between the environmental factor $\mathrm{n}$, shadowing factor $\eta$ and the signal strength in view of our proposed approach.

Originality/value: In our study, a methodology to calibrate the parameters of RSSI model is proposed with smaller errors. At the same time, three primary conventional model is evaluated based on the fitted regression.

Keywords: RSSI model, environmental factor n, shadowing factor $\eta$, intelligent position, experimental performance

\section{Introduction}

Wireless Sensor Networks (WSNs) (Davey, Jacobus, Namineni \& Siebert, 2010; Kamali, Laibinis, Petre \& Sere, 2014) have received considerable attention in the past few years. WSNs has made a great contribution to the technique of vehicle intelligent position systems. Throughout the experimental exploration as well as real-world tracking application, a large number of information collection data could be analyzed to optimize practicing transportation operation. For instance, a wireless sensor network for intelligent transportation system is proposed (Bohli, Hessler, Ugus \& Westhoff, 2008), road condition and the traffic state is acquired with ITS (Losilla, García-Sánchez, García-Sánchez \& García-Haro, 2012), the problem of adaptive traffic light control using real-time traffic information collected by a WSN is investigated (Zhou, Cao, Zeng \& Wu, 2010), as well as unmanned vehicle (Wan, Suo, Yan \& Liu, 2011), see Figure 1.

RSSI is a measurement to corresponding distance with signal strength of each pair of nodes. Multi-path fading due to scatters in the medium, shadowing effect, and a change in the antenna's radiation pattern may decrease the RSSI-based ranging accuracy (Stoyanova, Kerasiotis, Prayati \& Papadopoulos, 2007). A precisely optimized calibration for the RSSI model is a process of adjusting parameters, namely environmental factor $n$; shadowing factor $\eta$. The calibration is standardized, which is that experimental statistical data are comparison with real values. It simply depends on the average errors. Our object of this paper is to minimize the average errors and optimize the accuracy of RSSI model's parameters.

The most common exploration method is the fingerprint (Bahl \& Padmanabhan, 2000). The fingerprint, as an empirically comparative method, is to estimate the experimentally obtained distance between two sensor nodes with real value. A variant of the fingerprint method (Sun, Lang, Wang \& Liu, 2014) interpolates the measured data to give a better fitting on a RSSI value. Advanced statistical and signal processing methods to mitigate channel distortion and packet loss are utilized to achieve tracking resolution of few centimeters (Blumrosen, Hod, Anker, Dolev \& Rubinsky, 2010). 
The signal attenuation model is influenced by environmental factors tremendously and can measure distance through the signal attenuation considering primary environmental factors. Establishing accurate fitting degree and wide applicable scope is target for foreign and domestic researchers. Based on the wireless local area network equipments. Wang compared 13160 group data of errors in order to conclude that the distance error of linear and quadratic regression model is very large and cubic or more times regression model can reduce errors, so that he selected cubic regression model (Wang, Jia, Lee \& Li, 2003). Scott put forward Loss Prediction Model for Indoor Wireless Communications Multi-floored Buildings. Accordingly, considering the influence of obstacle and neglected the influence of flood, signal attenuation factor n (recommended value) is calculated (Seidel \& Rapport, 1992). Smailagic established quadratic objective function model with signal strength and distance from TMI approach (triangulation-based remapped interpolated approach) (Smailagic \& Kogan, 2002).

Bahl established WAF model (Wall Attenuation Factor), and determined the products of WAF and the number of obstacles between anchor nodes and target nodes (Bahl \& Padmanabban, 2000). Small established signal propagation model, considering composition of obstacles inside room and measurement errors and other factors on the day and night, finding that the model has high adaptability inside rooms (Small, 2000).

Great advantages of the above approaches described are their independence of the undisturbed underlying channel. However, it makes them difficult to reach a low localization error even in the presence of a good radio channel. With the drawbacks overcome by our proposed optimized strategy, we explore the parametric performances and determine the formulation of RSSI model. The straightforward method applications and diachronic formulations are key characteristics of theoretical innovations.

The paper is organized as follows: Section 1 introduces the literature about RSSI Model for the Vehicle Intelligent Position System. Section 2 describes the background and procedures of our experiment. In Section 3 the optimized algorithm is elaborated for minimized ranging errors. The implementation results of the experiment are analyzed in Section 4. The paper ends in Section 5 with conclusions. 


\section{Experiment}

\subsection{Background}

A key step in RSSI research methodology is the data collection phase (Bahl \& Padmanabhan, 2000). The RSSI-based tracking system includes calibration, range estimation, location estimation and refinement (Blumrosen, Hod, Anker, Dolev \& Rubinsky, 2010), which is the core concept of our experimental exploration.

In our experimental area, four anchor nodes ( $, B, C, D)$ are set to transmitting terminal and target nodes (blue nodes shown as Figure 2 ) are set to receiving end. It is limited to singlehop communication, so we can get all coordinates (104 coordinates) in Figure 2. Based on the coordinates, we can calculate the distance between anchor nodes and target nodes. What's more, we can see the nodes' distribution in Figure 3.

In a series of experiments, we located outside experimental area including obstacles, in which there is a tree on the anchor node B and a wall on the anchor node D. From Figure 2, we can see that variations between signal strength and distance are according to some rules. On the anchor node B and D, which are long-distance node, they appear some concentrated phenomenon when we are drawing nodes between signal strength and distance.

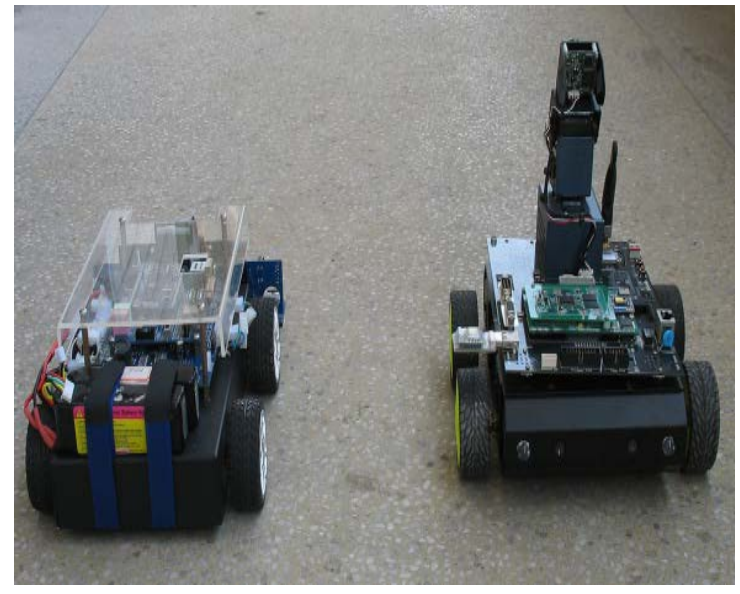

Figure 1. Intelligent vehicle with wireless sensors (Wan, Suo, Yan \& Liu, 2011)

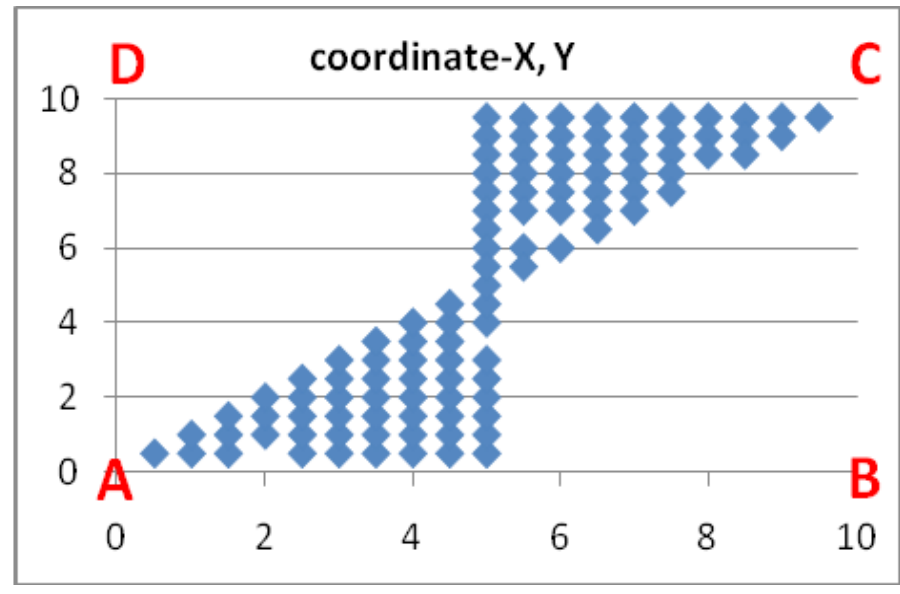

Figure 2. 104 experimental hopscotch coordinates

\subsection{Distance Estimation}

In order to achieve accurate positioning with WSN, a large number of localization algorithms are established, such as (Blumrosen, Hod, Anker, Dolev, \& Rubinsky, 2010; Wang, Jia, Lee \& Li, 2003; Seidel \& Rapport, 1992; Smailagic \& Kogan, 2002; Bahl \& Padmanabban, 2000; Seidel \& Rappaport, 1991; Hightower, Want \& Boriello, 2000). The distance between sensor nodes can be simply estimated according signal strength consumption. Multilateration algorithm is a familiar localization approach based on the measuring distance formulation. 
However, the target nodes need to be located by $\mathrm{n}(n \geq 3)$ anchors, at least three of which are no collinear. An ideal scenario of three anchors can be defined as Trilateration (Karl \& Willig, 2005). For the sake of minimized ranging errors, there are multiple $(n=4)$ anchor nodes to estimate the distance in our experiments. Multilateration approach calculates the positions of the target nodes $\left(x_{t}, y_{t}\right)$ by establishing an over determined system of Equation (1) (Karl \& Willig, 2005; Wang, Yuan, Laur \& Lang, 2011):

$$
\left\{\begin{array}{c}
\sqrt{\left(x_{t}-x_{1}\right)^{2}+\left(y_{t}-y_{1}\right)^{2}}=d_{1} \\
\sqrt{\left(x_{t}-x_{2}\right)^{2}+\left(y_{t}-y_{2}\right)^{2}}=d_{2} \\
\vdots \\
\sqrt{\left(x_{t}-x_{n}\right)^{2}+\left(y_{t}-y_{n}\right)^{2}}=d_{n} .
\end{array}\right.
$$

The index $d_{n}(i=1 \ldots n)$ denotes the estimated distance from anchor node $\left(x_{i}, y_{i}\right)$ to the target node $\left(x_{t}, y_{t}\right)$, shown as Figure 3 .

The way by adjusting parameter factors of RSSI model in order to find relationship between parameter factors and errors, we make error analysis between experimental distance and actual distance. As a result, we can achieve the goal of optimizing model and minimizing errors.

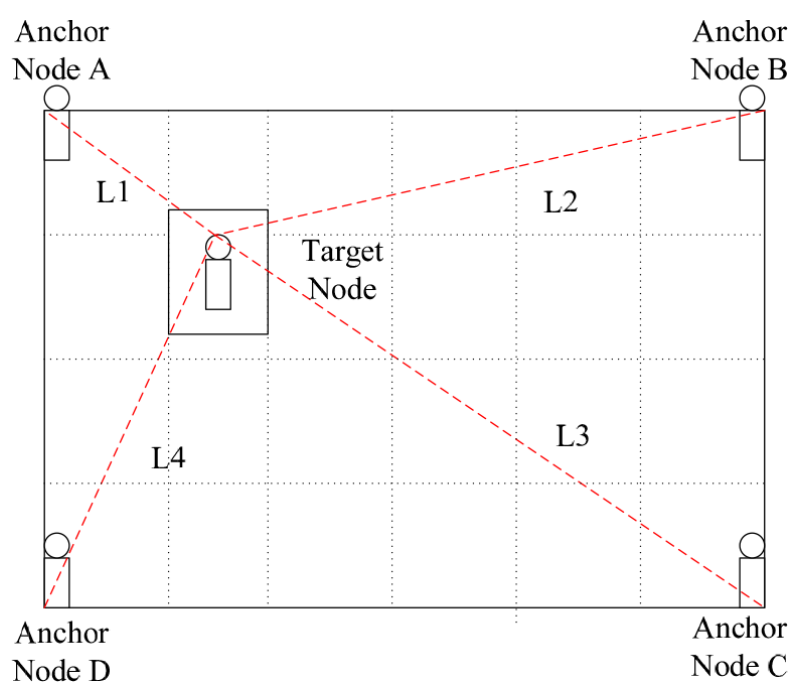

Figure 3. The principle of RSSI model 


\section{Model Formulation}

\subsection{Influencing Factors of RSSI}

Referring to sensors, signal strength is influenced not only by path length, but also by antenna's signal angle. What's more, the interference faced by the net of sensors is different in the various application environments. In the indoor area, wireless signal will be attenuated and even blacken-up by walls, floods, other obstacles and so on.

However, in the outdoor area, measured value of signal will produce errors as changing environmental conditions because of different environmental factors, such as pressure, humidity, temperature and so on.

Therefore, with environmental influencing and signal strength attenuation depending on the path, it is very important to establish and adjust the formulation for locating the target position precisely.

\subsection{Diachronic RSSI Model and Applied Environment}

\subsubsection{Log Path Loss Model}

Generally, the formulation of log path loss model is expressed as below:

$$
P l(d)=P l\left(d_{0}\right)+10 n \log _{10}\left(\frac{d}{d_{0}}\right)+x_{0} .
$$

In the Eq. (2), n means path loss factor, which is related with the environment, expressing the velocity of signal loss as distance increasing, and it is rang from 2 to $5 . x \sigma$ donates log normal distribution random variable, whose mean value is 0 and standard deviation is $2 . d_{0}$ donates reference range, which is Im generally. $P I\left(d_{0}\right)$ is expressed as the received frequency since their distance is 1 meter. $d$ is the distance between transmitting terminal and receiving end. The formulation of signal strength in the receiving end:

$$
P_{i}(d)=P_{t}(d)-P l(\mathrm{~d}) .
$$

Among them, $P_{i}(d)$ donates the received frequency, which is calculated by the signal strength. $P_{t}\left(d_{0}\right)$ is the sending frequency. $P I(d)$ is the frequency loss along with signal propagation. If the sending frequency is known, we can measure the received frequency and calculate the degree of signal loss by using Equation (2), as well as the distanced based on the Equation (3).

Log path loss model is aiming at nodes position adaptation in the outdoor wireless net. It can analyze radio wave propagation of path-loss in field environment. This approach pays less attention at the process of nodes commutation and position. What's more, its accurate positioning meets the regularly utilized requirements. 


\subsubsection{Attenuation Factor Model}

FAF in attenuation factor model will be discrepant in the various areas, which means it is appropriate for all kinds of circumstances. This model is always signal attenuation model. Standard deviation between estimated of signal attenuation and actual measurement of this one is $4 \mathrm{dBm}$, the formulation of attenuation factor model is shown below:

$$
\overline{P L}=\overline{P L}\left(d_{0}\right)+10 n_{\mathrm{SF}} \log _{10}\left(\frac{d}{d_{0}}\right)+F A F .
$$

Among them, $n_{S F}$ is index value of signal attenuation in the same layers. FAF donates flood attenuation factor.

\subsubsection{Matt MaEnNa Model}

The formulation of Matt MaEnNa model is as followings:

$$
L_{\text {pico }}=L_{\mathrm{o}}+10 n \log _{10}(\mathrm{~d})+\sum_{\mathrm{i}=1}^{J} N_{\mathrm{wj}} L_{\mathrm{wj}}+\sum_{\mathrm{i}=1}^{J} N_{\mathrm{fi}} L_{\mathrm{fi}}
$$

Among them, $L_{\text {pico }}$ is path-loss, $L_{o}$ is loss when the distance of reference point is $/$ meter, $n$ is index value of signal attenuation, $d$ is the distance between transmitting terminal and receiving end, $N_{w j}$ or $N_{f i}$ is the number of floods or walls in different styles through which sending signal is passing. $L_{w j}$ or $L_{f i}$ is the attenuation factor of flood or wall in different styles.

Matt MaEnNa model is suitable for the signal propagation in the buildings. In the different floors and rooms of buildings, floors and walls will be major attenuation factor, so the model is proper for indoor condition.

\subsubsection{Simplified Model}

$$
R S S I=-\left(10 n \log _{10} \mathrm{~d}+A\right) .
$$

In the formulation, $n$ is the index value of signal attenuation, and $A$ donates the received signal strength on reference range, which is $1 \mathrm{~m}$.

The simplified model applied in widely conditions, no matter indoor or outside, and it is utilized as a referred model in our experiments. Limiting conditions are less, so positioning accuracy of the model is lower.

\subsection{Comparison of RSSI Model}

From the diachronic models, the basic forms of RSSI model: the signal strength of target node is dependent variable, and the distance $d$ defined from target nodes to anchor nodes is dependent variable, such as $R S S I=f(d)$. Based on the experimental results, the fitting degree of linear, index, power and other functions is low, besides their error is big. Log and polynomial function-model have very good value for our study. 
With our experiments conducted, we can acquire the experimental data to which the distances of different positions at anchor nodes and signal strength are related. In the Figure 4 and 5, the distances between anchor node $A$ and target node are defined as abscissa, and the signal strength is used as ordinate. The formulations and fitting degrees are abstracted from log and polynomial function-model in the Figure 4 and 5.

\subsubsection{Log Model}

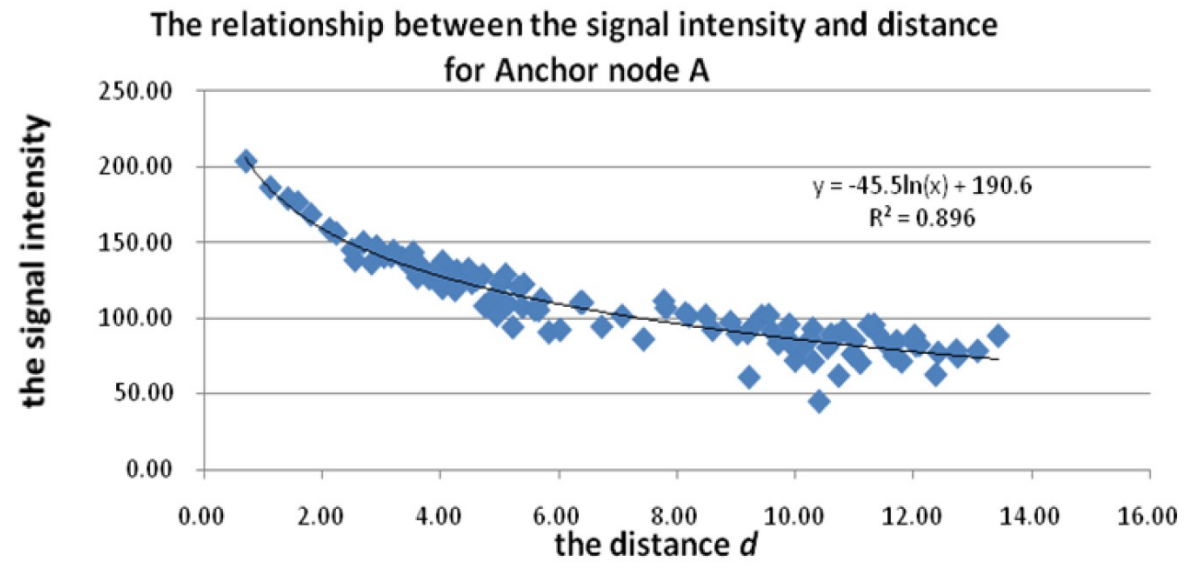

Figure 4. Log model

Based on Figure 4, fitting degree of log function is 0.896 , this value is very high, as the same time, environmental factors $n=4.55$, whose diachronic value is rang from 2 to 5 , so it is very ideal fitting model.

In order to match fitting model and tracing point data, we need optimize log model, adjust environmental factor $\mathrm{n}$ and shadowing factor $n$ to reduce errors in mathematical approach, so the trend of $n, \eta$ with errors can be fined.

\subsubsection{Polynomial Model}

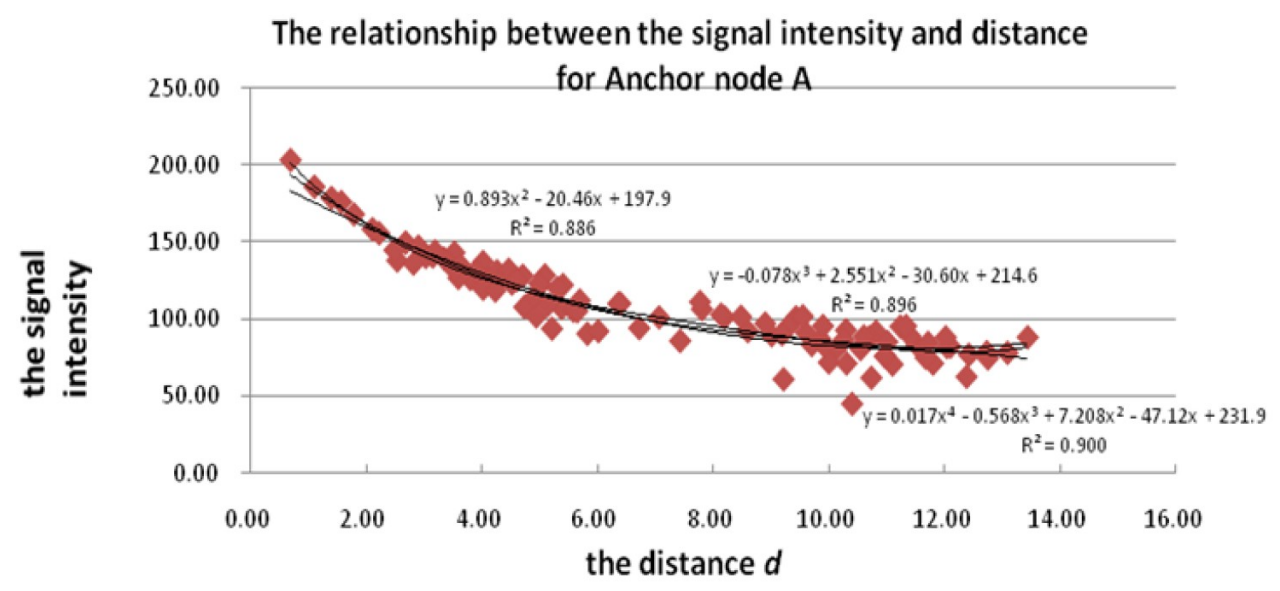

Figure 5. The polynomial model 
Shown as Figure 5, in a great deal of fitting experiments, the fitting degree is increasing as trend line-function's times are increasing in the polynomial model. However, when the degree of polynomial is higher than 4 , the fitting degree will maintain 0.900 and change no more. As the fitting degree is a small different between the degrees of polynomial 3 (higher than 4) and 4 , we determine cubic polynomial as analytical foundation.

Lagrange interpolation, and used signal area are applied as plane grid to form many Lagrange polynomial curves (Zhu, Zhou \& Ma, 2009). In addition, the researchers continuously interpolated so as to reduce errors. This approach applied into indoor closed small scale and its adaptability and robustness is relatively worse, besides that the mathematics algorithm is very complicated. As a result, the polynomial model is unusually used as benchmark model to analyze.

\subsubsection{Errors Comparison Between Log Model and Polynomial Model}

The formulation of log model fitted for the first time:

$$
R S S I=-45.5 \ln \mathrm{d}+190.6 .
$$

The polynomial model as quadratic polynomial mode:

$$
R S S I=0.017 \mathrm{~d}^{4}-0.568 \mathrm{~d}^{3}+7.28 \mathrm{~d}^{2}-47.12 \mathrm{~d}+231.9 \text {. }
$$

The fluctuation of both log model and polynomial one involved in the real signal strength at the same distances is described as Figure 6:

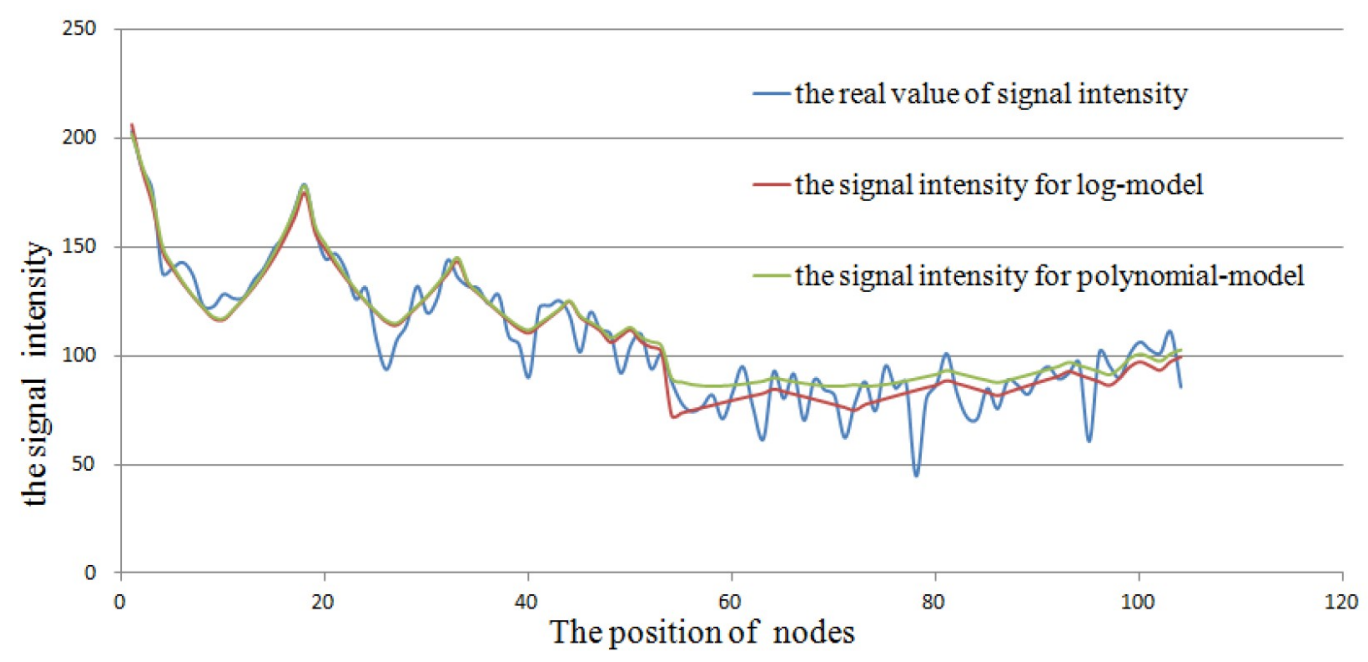

Figure 6. Fluctuation of two kinds of model

This is considered within the average errors in which the values of the log model and the polynomial model are $7.99 \%$ and $8.66 \%$, respectively. We regard the major drawback of the polynomial model as the difficulty of error reduction. What's more, the factors of the 
polynomial model are so many that it is very confused to clarify the definitions of parameters and improve them.

\subsection{The Relationship Between Influence Factors and Errors in RSSI Model}

\subsubsection{Parameter Calibration}

Based on the diachronic model and fitting model from experimental data, a simplified formulation of nodes-distance and signal strength can be determined:

$$
\begin{gathered}
R S S I=-10 n \ln \mathrm{d}+\eta . \\
\eta=\operatorname{Pt}(d)-\operatorname{Pl}\left(d_{0}\right)-x_{0} .
\end{gathered}
$$

Among them, $n$, being quantified, donates environmental factor (or path-loss factor), $d$ is the distance between transmitting terminal and receiving end, $\operatorname{Pt}(d)$ is the frequency from transmitting terminal, $P I\left(d_{0}\right)$ is the frequency as the distance is $1 \mathrm{~m}, x \sigma$ is shadowing factor. According to experience, the mean value of $x \sigma$ is 0 and the variation range of $x \sigma$ is $4 \sim 10$.

\subsubsection{The Relationship Between Environmental Factors $n$ and Errors}

There are four anchor nodes and one target node in this experiment. We can obtain 4 groups data besides distance and corresponding signal strength. In the section 3, this issue chooses one group data (anchor A) 104 pairs to introduce. The fitting formulation from analyses:

$$
R S S I=-45.5 \ln \mathrm{d}+190.6 .
$$

Or

$$
y=-45.5 \ln x+190.6 .
$$

That means $n=4.55, \eta=190.6$.

\subsubsection{Analysis Process of Errors}

From formulation (11), we can see:

$$
\mathrm{d}=e^{\frac{\eta-R S S I}{10 \mathrm{n}}}
$$

For example, we will elaborate the analysis process of data from the anchor node A:

- As $\eta=190.6$, and $n=2 \sim 5$, we will enlarge $n$ studying-range from 1 to 10 . As $n$ is ranged from 1 to10, we can obtain $104 \times 10$ matrices for distance data based on that signal frequency RSSI substitutes formulation (13).

- A s $n=1,2, \cdots, 10$, we can get the errors from the subtracting between the experimental data and the real data. As a result, 10 accumulated errors can be generated. 
- We choose 3 groups of smallest accumulated errors $(n=4,5,6)$, and utilize the mathematic approaches of successive approximation. As $n=4.1,4.2, \cdots, 5.9$ we will use those 19 values and repeat approach(i), (ii), to obtain 19 accumulated error values.

- We choose 3 groups of smallest accumulated errors $(n=4.5,4.6,4.7)$, and use the mathematic approaches of successive approximation. As $n=4.51,4.52, \cdots, 4.69$, we use those 19 values and repeat approach (i), (ii). Therefore, 19 accumulated error values are acquired and $n=4.58$ is derived as the optimal result.

- From the exploration of data and the function expression, the trend of between $n$ and errors is parabola mode. Finally, as $n=4.58$, error value is smallest. What's more, the environmental factor in the optimized model is $n_{1}=4.58$.

- In the same way, repeated the approach (iii), (iv), (v) for anchor nodes B, C, D. As a result, we can know $n_{2}=4.82, n_{3}=4.26, n_{4}=5.44$ as the same optimal values.

\section{Experiments' Evaluation}

\subsection{The Relationship Between Environmental Factor $\boldsymbol{n}$ and Error}

Figure 7 shows the trend of $\mathrm{n}$ and error, as environmental factornand mean error is defined as abscissa and ordinate, respectively.

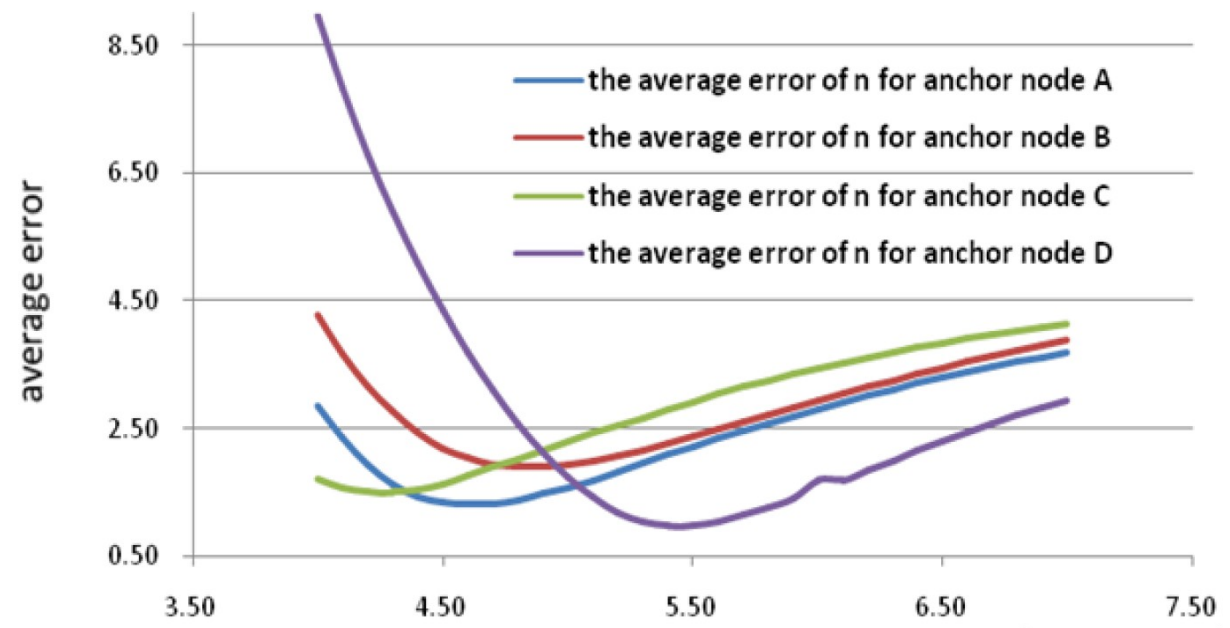

The environmental factor $\mathrm{n}$ in the models based on the anchor nodes ( $\mathrm{A}, \mathrm{B}, \mathrm{C} 、 \mathrm{D}$ )

Figure 7. The formulation-relationship between $\mathrm{n}$ and error

From the Figure 7, we can see the trend is a parabola function. As $\mathrm{n}$ is determined as a certain value, the mean error of the parabolic function is smallest. 


\subsection{The Relationship Between Shadowing Factor $\eta$ and Error}

As the shadowing factor $\eta$ is abscissa and the mean error is ordinate, the trend of $\eta$ and error is illustrated in Figure 8.

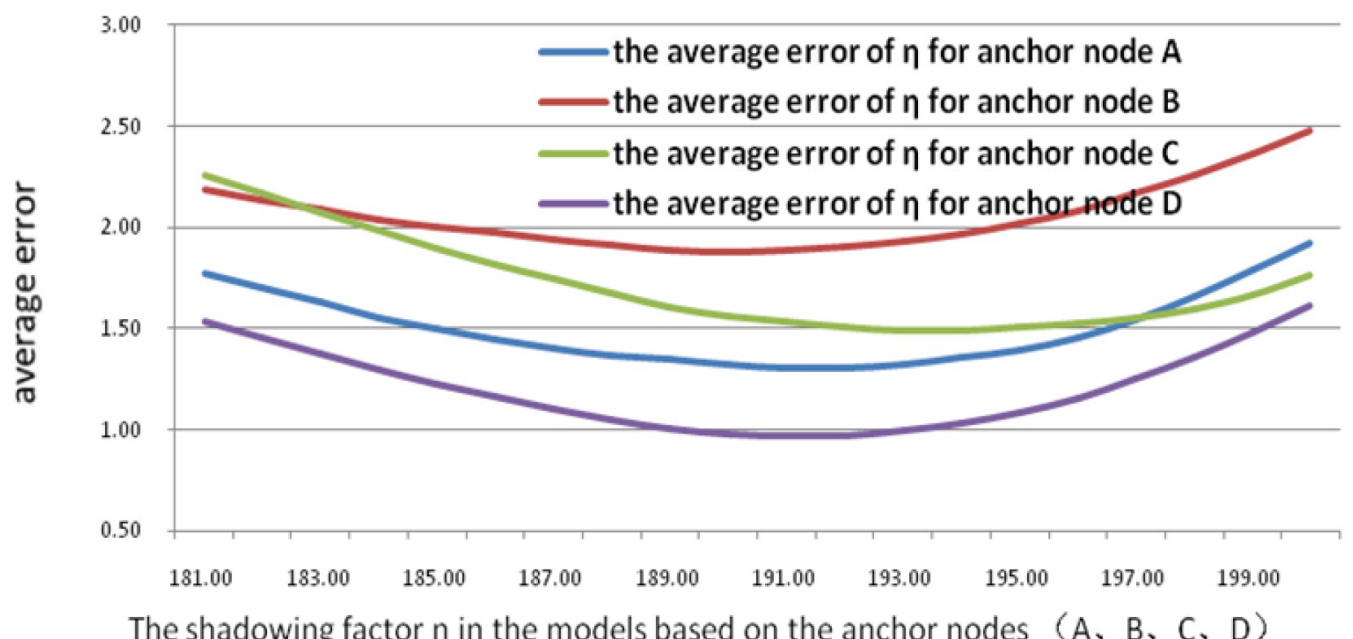

Figure 8. The formulation-relationship betweennand error

From the Figure 8 , we can see the trend is parabola function. As $\eta$ is a certain value, the mean error is smallest, so it proves that RSSI model has been optimized.

\subsection{The Discussion of Factors $n, \eta$ in RSSI Model}

\subsubsection{RSSI Model Optimization}

Based on the above optimization approaches, the anchor node A fitting description from the initial point formulation (11) is optimized for:

$$
R S S I=-45.8 \ln \mathrm{d}+191
$$

We plug the frequency value RSSI of signal strength of anchor node A that received from the destination node into formulation (14). Base on the experimental data of anchor node $A$ and destination node's distance, the average error of $16.62 \%$ is determined by comparing with the actual distance. The average error of the first described point fitting formulation (11) is $16.74 \%$, which means the error is reduced and a more optimized result appears.

With the RSSI model of Anchor node (A, B, C, D) optimized, the average error is reduced from $18.79 \%$ to $18.22 \%$. 


\subsubsection{The Relationships Among Environmental Factors $n$, Shadowing Factor $\eta$ and Distance $d$}

In the RSSI model, environmental factors $n$, shadowing factor $\eta$, can be changed because of such situations as the environment, experimental equipment, etc. Through fitting models based on experimental data, we will further explore the errors generated by the models in the different ranges of distance $d$ such as the short distance, middle distance, long-distance. Then we explore the relationships among the environmental factors $n$, shadowing factor $\eta$ and distance $d$. as shown in Figure 9.

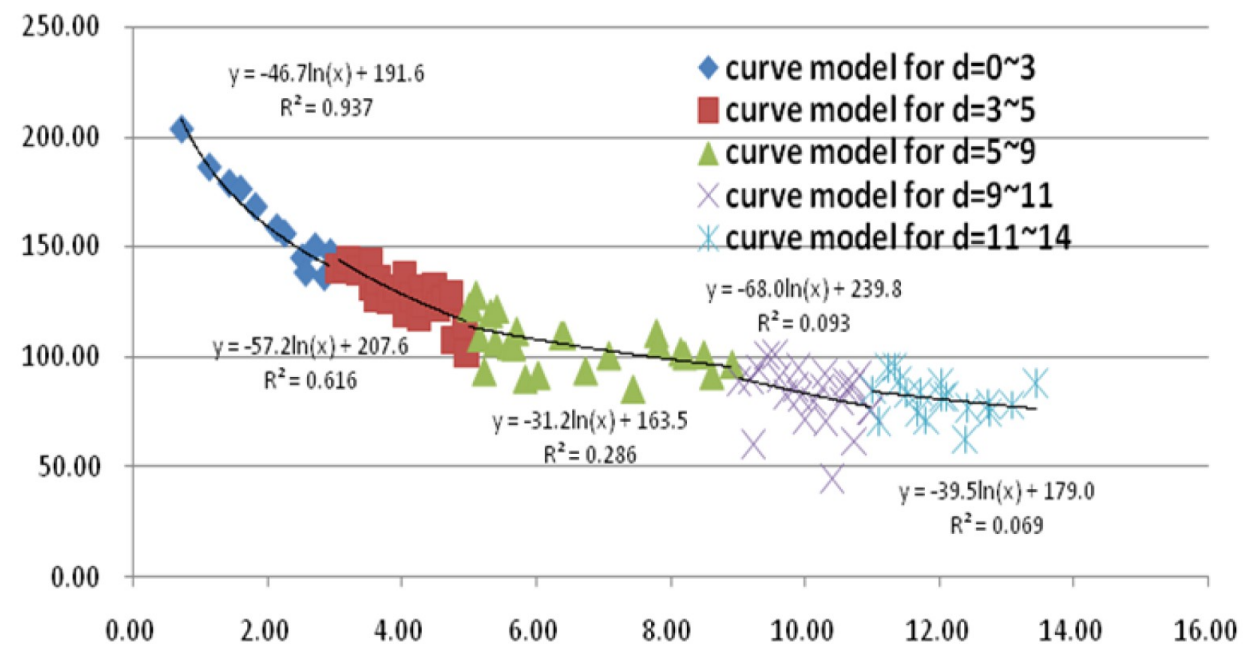

Figure 9. The fitting models of different distance ranges

According to the different ranges of distance $d$, the five kinds of model are fitted, and the values of environment factors $n$, shadowing factor $\eta$ are shown as Table 1 :

\begin{tabular}{|l|c|c|c|c|c|}
\hline Signal distance $\boldsymbol{d}$ & $\mathbf{0 - 3}$ & $\mathbf{3 - 5}$ & $\mathbf{5 - 9}$ & $\mathbf{9 - 1 1}$ & $\mathbf{1 1 - 1 4}$ \\
\hline Environmental factor $\boldsymbol{n}$ & 4.67 & 5.72 & 3.12 & 6.8 & 3.95 \\
\hline Shadowing factor $\boldsymbol{\eta}$ & 191.6 & 207.6 & 163.5 & 239.8 & 179 \\
\hline Location error & $9.46 \%$ & $9.23 \%$ & $26.95 \%$ & $13.92 \%$ & $16.22 \%$ \\
\hline
\end{tabular}

Table 1. Fitting model in short distance

Establish the three-dimensional coordinate's relationship between the environmental factor $n$, the shadowing factor $\eta$ and the location error, as shown in Figure 10. 


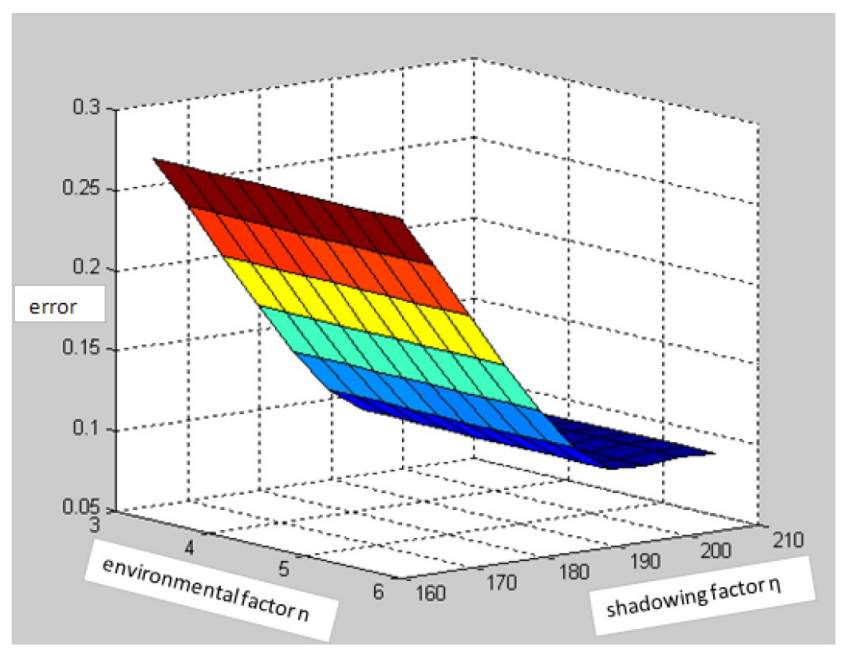

Figure 10. Three-dimensional coordinate graph basing on Figure 1

From the analysis of Table1 and Figure 9, we can conclude that there exist a certain increasing trend when the environmental factor $n$ increases with the distance $d$, but after the middle distance $(d \geq 5 \mathrm{~m}$ ), error is extremely increased, so the increasing trend become to fluctuate. Shadowing factor $\eta$ will also perform a certain increasing trend with the signal distance $d$ increased, there also are the same fluctuations from the environmental factors.

According to this principle, the signal distance $d$ cumulative way model comparison, the establishment of Table 2, as follows:

\begin{tabular}{|l|c|c|c|c|c|}
\hline Signal distance $\boldsymbol{d}$ & $\mathbf{0 - 3}$ & $\mathbf{0 - 5}$ & $\mathbf{0 - 9}$ & $\mathbf{0 - 1 1}$ & $\mathbf{0 - 1 4}$ \\
\hline Environmental factor $\boldsymbol{n}$ & 4.67 & 4.8 & 4.55 & 4.72 & 4.55 \\
\hline Shadowing factor $\boldsymbol{\eta}$ & 191.6 & 193.5 & 190.7 & 192.8 & 190.6 \\
\hline Location error & $9.46 \%$ & $10.72 \%$ & $14.52 \%$ & $16.19 \%$ & $16.75 \%$ \\
\hline
\end{tabular}

Table 2. Fitting model in continuous distance

With the cumulative increase of signal distance $d$, the location error increases, the variation of environmental factor $n$, shadowing factor $\eta$ has slightly increased trend, and with certain fluctuation. Therefore, this paper makes the following assumptions: at a distance $d$ from the near to the distant, the environmental factor (or shadowing factor) $\eta$ of the model will correspondingly increase in the trend. 


\subsubsection{The Relationship Among Environmental Factor $n$, Shadowing Factor $\eta$ and Signal Strength}

Depending on the signal strength, we build a fitting model respectively in the range of signal variation from low to high. Analyzing the error, the changing trend of the environmental factor $\mathrm{n}$, shadowing factor nand signal strength is shown in Figure 11.

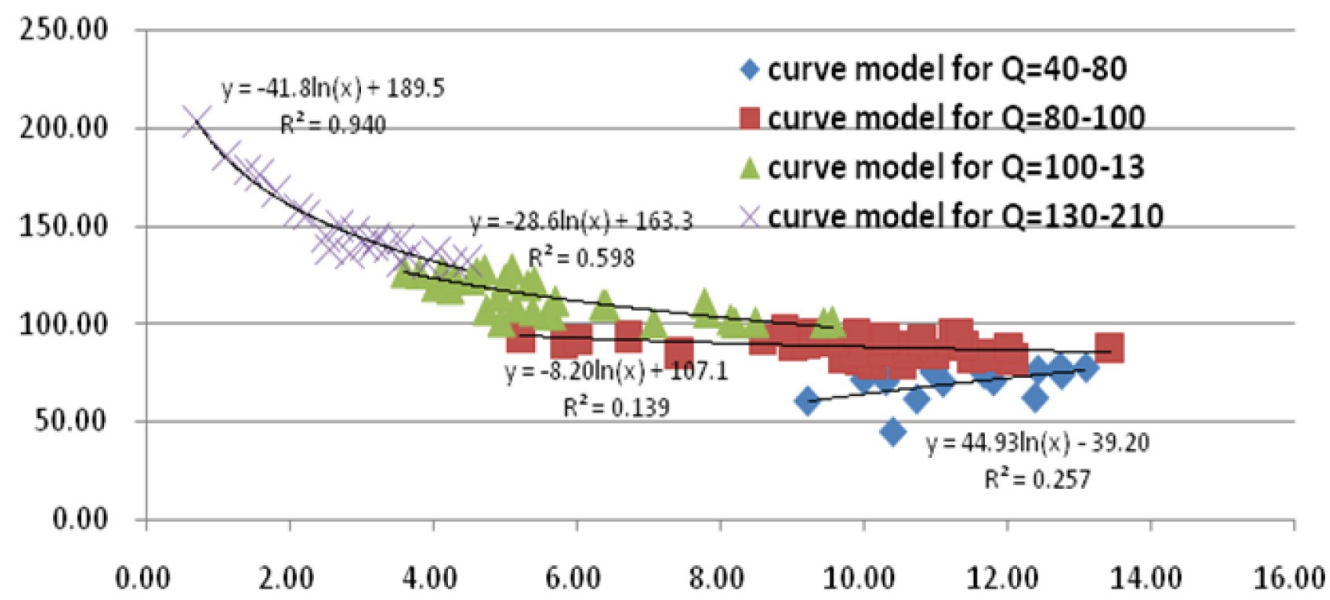

Figure 11. Fitting model of signal strength in different distance

\begin{tabular}{|l|c|c|c|c|c|}
\hline Signal distance $\boldsymbol{d}$ & $\mathbf{4 0 - 8 0}$ & $\mathbf{8 0 - 1 0 0}$ & $\mathbf{1 0 0 - 1 3 0}$ & $\mathbf{1 3 0 - 2 1 0}$ & $\mathbf{4 0 - 8 0}$ \\
\hline Environmental factor $\boldsymbol{n}$ & 4.18 & 2.86 & 0.82 & -4.49 & 4.18 \\
\hline Shadowing factor $\boldsymbol{\eta}$ & 189.5 & 163.3 & 107.1 & -39.2 & 189.5 \\
\hline Location error & $61.33 \%$ & $44.77 \%$ & $88.25 \%$ & - & $61.33 \%$ \\
\hline
\end{tabular}

Table 3. Fitting model in long distance

According to Figure 11, the four fitting models' parameters and error with the range of signal strength from low to high is shown in Table 3.

From the above table, we can find the error is very large, so the implicit relation between fitting environmental factors $\mathrm{n}$ and shadowing factor $\eta$ has been presented. 


\section{Conclusions and Future Work}

In this paper, we analyzed various main diachronic formulations of RSSI model. Through comparing the random errors for those models, the parameters of those models are demarcated, using the real data from four different scenarios of our experiments (shown as appendix list at the end of paper). For different formulations, the degree of fitting is analyzed in detail as follows:

1. Polynomial model is an important branch in the development of the signal status model, but the error is much bigger than in the logarithm model. Not only the approach of improving the precision is very complex, but also the physical meaning is obscure in view of the form of the model itself, thus it is considered as the main disadvantages of existing polynomial model in our paper.

2. Because there are errors between the actual data and the calculated results from selected formulations, the optimizing RSSI model makes a great contribution to the accuracy position of the running vehicle. It plays a vital role on reducing the errors and optimizing the fitted results by exploring the rules between the errors and the environment factor $n$ and shadowing factor $\eta$ respectively, so as to conclude the reasonable parabolic function relation between them. As a result, a large number of experimental data is indispensable using wireless sensor networks to support intelligent transportation systems.

3. This paper describes in detail in exploring the function relationships between the errors and environment factor $\mathrm{n}$ and shadowing factor $\eta$ respectively, seeking the $\eta$ and $n$ to minimize the error, thus obtaining the fundamental optimization RSSI model. But the precision of this model is not so perfect compared with the original model. This is because the collection of the original data is inaccurate and the confluence point is of large degree of discrete as well as the large variation of the signal strength between two confluence points especially when they are in large distance.

4. The positioning error is on the increase according with the increase of signal distance $d$. The most important parameters (environmental factor $n$, shadowing factor $\eta$ ) can be expressed on a trend of fluctuation increase with the increase of distance $d$. However, when it is in certain signal strength, the fitting error of the model is very big but the change rule of environmental factor $n$, shadowing factor $\eta$ and the signal strength is not obvious no matter the signal strength is high or low.

5. The advantage of our study is that the accuracy of RSSI model can be optimized through our simple proposed approaches. In our paper, the changing rules of the factors in the signal attenuation model are researched and the physical definition of the parameters is clarified. Additionally, we failed to find the variation trend between the environmental factor $n$, shadowing factor $\eta$ and the signal strength in view of the method. 


\section{Acknowledgements}

This paper is supported by "National Basic Research Program of China (No. 2012CB725403)".

\section{References}

Bahl, P., \& Padmanabhan, V.N. (2000). RADAR: An in-building RF-based user location and tracking system. Nineteenth Annual Joint Conference of the IEEE Computer and Communications Societies. Proceedings, 2(1), 775-784. http://ieeexplore.ieee.org/xpls/abs_all.jsp? arnumber $=832252 \& \operatorname{tag}=1$

Blumrosen, G., Hod, B., Anker, T., Dolev, D., \& Rubinsky, B. (2010). Continuous close-proximity rssi-based tracking in wireless sensor networks. Body Sensor Networks, 5(1), 234-239. http://ieeexplore.ieee.org/xpls/abs_all.jsp?arnumber $=5504760$

Bohli, J.M., Hessler, A., Ugus, O., \& Westhoff, D. (2008). A secure and resilient WSN roadside architecture for intelligent transport systems. The first ACM conference on Wireless network security, 55(1), 161-171. http://dx.doi.org/10.1145/1352533.1352562

Davey, T., Jacobus, C.J., Namineni, P.K., \& Siebert, G. (2010). Wireless mobile indoor/outdoor tracking system. U.S. Patent, 852(7), 262-265. http://www.google.com/patents/US7852262

Hightower, J., Want, R., \& Borriello, G. (2000). SpotON: An indoor 3D location sensing technology based on RF signal strength. Department of Computer Science and Engineering, Seattle, WA, 1(1), 4-14. http://ahvaz.ist.unomaha.edu/azad/temp/sal/00-hightower-localization-indoor-3drssi-spoton-techrep.pdf

Kamali, M., Laibinis, L., Petre, L., \& Sere, K. (2014). Formal development of wireless sensor-actor networks. Science of Computer Programming, 80(1), 25-49.

http://dx.doi.org/10.1016/j.scico.2012.03.002

Karl, H., \& Willig, A. (2005). Protocols and architectures for wireless sensor networks. England: John Wiley \& Sons. http://dx.doi.org/10.1002/0470095121

Losilla, F., García-Sánchez, A.J., García-Sánchez, F., \& García-Haro, J. (2012). On the role of wireless sensor networks in intelligent transportation systems. Transparent Optical Networks, 14(1), 1-4. http://ieeexplore.ieee.org/xpls/abs_all.jsp?arnumber=6253846\&tag=1

Seidel, S.Y., \& Rapport, T.S. (1992). 914 MHz Path Loss Prediction Model for Indoor Wireless Communications Multi-floored Buildings. IEEE Transactions on Antennas a Propagation, 40(2), 207-217. http://dx.doi.org/10.1109/8.127405

Seidel, S.Y., \& Rappaport, T.S. (1991). $900 \mathrm{MHz}$ path loss measurements and prediction techniques for in-building communication system design. Vehicular Technology Conference, 41(1), 613-618. http://ieeexplore.ieee.org/xpls/abs_all.jsp?arnumber=140565

Smailagic, A., \& Kogan, D. (2002). Location sensing and privacy in a context-aware computing environment. Wireless Communications, 9(5), 10-17. http://dx.doi.org/10.1109/MWC.2002.1043849 
Stoyanova, T., Kerasiotis, F., Prayati, A., \& Papadopoulos, G. (2007). Evaluation of impact factors on RSS accuracy for localization and tracking applications. Proceedings of the 5th ACM international workshop on Mobility management and wireless access, 5(1) , 9-16. http://dx.doi.org/10.1145/1298091.1298094

Sun, Y., Lang, M., Wang, D., \& Liu, L. (2014). A PSO-GRNN model for railway freight volume prediction: Empirical study from China. Journal of Industrial Engineering and Management, 7(2), 413-433. http://dx.doi.org/10.3926/jiem.1007

Wan, J., Suo, H., Yan, H., \& Liu, J. (2011). A general test platform for cyber-physical systems: unmanned vehicle with wireless sensor network navigation. Procedia Engineering, 24(1), 123-127. http://dx.doi.org/10.1016/j.proeng.2011.11.2613

Wang, X., Yuan, S., Laur, R., \& Lang, W. (2011). Dynamic localization based on spatial reasoning with RSSI in wireless sensor networks for transport logistics. Sensors and Actuators A: Physical, 171(2), 421-428. http://dx.doi.org/10.1016/j.sna.2011.08.015

Wang, Y., Jia, X., Lee, H.K., \& Li, G.Y. (2003). An indoors wireless positioning system based on wireless local area network infrastructure. Satellite Navigation Technology Including Mobile Positioning \& Location Services, 54(6), 359-365.

http://www.sage.unsw.edu.au/snap/publications/wangy_etal2003a.pdf

Zhou, B., Cao, J., Zeng, X., \& Wu, H. (2010). Adaptive traffic light control in wireless sensor network-based intelligent transportation system. Vehicular Technology Conference Fall, 72(1), 1-5. http://ieeexplore.ieee.org/xpls/abs_all.jsp?arnumber=5594435

Zhu, J., Zhou, S, \& Ma, Q. (2009). An indoor localization algorithm based RFID. Control and Automation Publication Group, 82(25), 160-162.

http://www.cnki.com.cn/Article/CJFDTotal-WJSJ200923068.htm 


\section{Appendix A}

\section{Experimental data attached list}

\begin{tabular}{|c|c|c|c|c|c|c|c|c|c|}
\hline \multicolumn{2}{|c|}{ Coordinate } & \multicolumn{4}{|c|}{$\begin{array}{l}\text { Signal strength between } \\
\text { target node and anchor node }\end{array}$} & \multicolumn{4}{|c|}{$\begin{array}{l}\text { Real distance between } \\
\text { target node and anchor node }\end{array}$} \\
\hline$x$ & $\mathbf{Y}$ & $\mathbf{A}$ & B & C & D & $\mathbf{A}$ & B & C & D \\
\hline 0.5 & 0.5 & 203.33 & 83.67 & 79.00 & 74.67 & 0.71 & 9.51 & 13.44 & 9.51 \\
\hline 1 & 0.5 & 186.00 & 102.33 & 96.00 & 98.33 & 1.12 & 9.01 & 13.09 & 9.55 \\
\hline 1.5 & 0.5 & 176.00 & 102.00 & 99.33 & 91.67 & 1.58 & 8.51 & 12.75 & 9.62 \\
\hline 2.5 & 0.5 & 138.00 & 93.00 & 96.00 & 93.00 & 2.55 & 7.52 & 12.10 & 9.82 \\
\hline 3 & 0.5 & 140.33 & 95.33 & 74.00 & 102.67 & 3.04 & 7.02 & 11.80 & 9.96 \\
\hline 3.5 & 0.5 & 143.00 & 99.00 & 87.00 & 75.00 & 3.54 & 6.52 & 11.51 & 10.12 \\
\hline 4 & 0.5 & 137.00 & 89.33 & 52.67 & 83.67 & 4.03 & 6.02 & 11.24 & 10.31 \\
\hline 4.5 & 0.5 & 123.00 & 98.67 & 87.33 & 64.00 & 4.53 & 5.52 & 10.98 & 10.51 \\
\hline 5 & 0.5 & 122.67 & 129.67 & 78.00 & 95.33 & 5.02 & 5.02 & 10.74 & 10.74 \\
\hline 5 & 1 & 128.33 & 112.00 & 82.67 & 90.33 & 5.10 & 5.10 & 10.30 & 10.30 \\
\hline 4.5 & 1 & 126.33 & 108.00 & 86.67 & 84.00 & 4.61 & 5.59 & 10.55 & 10.06 \\
\hline 4 & 1 & 127.00 & 108.33 & 93.33 & 88.00 & 4.12 & 6.08 & 10.82 & 9.85 \\
\hline 3.5 & 1 & 135.00 & 118.00 & 86.00 & 87.00 & 3.64 & 6.58 & 11.10 & 9.66 \\
\hline 3 & 1 & 140.67 & 108.00 & 84.67 & 94.00 & 3.16 & 7.07 & 11.40 & 9.49 \\
\hline 2.5 & 1 & 149.67 & 92.00 & 84.33 & 88.00 & 2.69 & 7.57 & 11.72 & 9.34 \\
\hline 2 & 1 & 155.67 & 109.00 & 89.00 & 65.00 & 2.24 & 8.06 & 12.04 & 9.22 \\
\hline 1.5 & 1 & 168.00 & 80.33 & 75.00 & 102.00 & 1.80 & 8.56 & 12.38 & 9.12 \\
\hline 1 & 1 & 178.67 & 72.33 & 46.67 & 95.00 & 1.41 & 9.06 & 12.73 & 9.06 \\
\hline 1.5 & 1.5 & 158.33 & 89.67 & 69.33 & 98.33 & 2.12 & 8.63 & 12.02 & 8.63 \\
\hline 2 & 1.5 & 144.67 & 98.67 & 90.00 & 77.67 & 2.50 & 8.14 & 11.67 & 8.73 \\
\hline 2.5 & 1.5 & 147.00 & 65.00 & 83.00 & 77.00 & 2.92 & 7.65 & 11.34 & 8.86 \\
\hline 3 & 1.5 & 139.33 & 93.33 & 77.00 & 96.33 & 3.35 & 7.16 & 11.01 & 9.01 \\
\hline 3.5 & 1.5 & 126.00 & 95.33 & 85.67 & 73.67 & 3.81 & 6.67 & 10.70 & 9.19 \\
\hline 4 & 1.5 & 131.00 & 103.67 & 83.00 & 99.67 & 4.27 & 6.18 & 10.40 & 9.39 \\
\hline 4.5 & 1.5 & 107.67 & 109.67 & 90.00 & 97.67 & 4.74 & 5.70 & 10.12 & 9.62 \\
\hline 5 & 1.5 & 93.67 & 114.00 & 91.00 & 87.33 & 5.22 & 5.22 & 9.86 & 9.86 \\
\hline 5 & 2 & 107.00 & 116.00 & 94.00 & 100.00 & 5.39 & 5.39 & 9.43 & 9.43 \\
\hline 4.5 & 2 & 114.67 & 92.00 & 107.33 & 101.67 & 4.92 & 5.85 & 9.71 & 9.18 \\
\hline 4 & 2 & 132.00 & 99.67 & 78.00 & 93.00 & 4.47 & 6.32 & 10.00 & 8.94 \\
\hline 3.5 & 2 & 119.67 & 99.33 & 84.00 & 86.67 & 4.03 & 6.80 & 10.31 & 8.73 \\
\hline 3 & 2 & 126.67 & 103.00 & 96.67 & 90.67 & 3.61 & 7.28 & 10.63 & 8.54 \\
\hline 2.5 & 2 & 144.00 & 102.00 & 85.00 & 103.00 & 3.20 & 7.76 & 10.97 & 8.38 \\
\hline 2 & 2 & 136.00 & 113.67 & 72.67 & 89.67 & 2.83 & 8.25 & 11.31 & 8.25 \\
\hline 2.5 & 2.5 & 132.00 & 102.67 & 96.67 & 88.33 & 3.54 & 7.91 & 10.61 & 7.91 \\
\hline 3 & 2.5 & 131.00 & 101.00 & 57.00 & 90.00 & 3.91 & 7.43 & 10.26 & 8.08 \\
\hline 3.5 & 2.5 & 124.00 & 81.67 & 112.00 & 96.33 & 4.30 & 6.96 & 9.92 & 8.28 \\
\hline 4 & 2.5 & 128.00 & 94.67 & 72.00 & 89.67 & 4.72 & 6.50 & 9.60 & 8.50 \\
\hline 4.5 & 2.5 & 109.00 & 108.00 & 89.00 & 92.00 & 5.15 & 6.04 & 9.30 & 8.75 \\
\hline 5 & 2.5 & 105.33 & 105.33 & 80.67 & 104.33 & 5.59 & 5.59 & 9.01 & 9.01 \\
\hline 5 & 3 & 90.33 & 99.00 & 105.00 & 95.33 & 5.83 & 5.83 & 8.60 & 8.60 \\
\hline 4.5 & 3 & 122.00 & 105.33 & 74.67 & 94.00 & 5.41 & 6.26 & 8.90 & 8.32 \\
\hline
\end{tabular}




\begin{tabular}{|c|c|c|c|c|c|c|c|c|c|}
\hline \multicolumn{2}{|c|}{ Coordinate } & \multicolumn{4}{|c|}{$\begin{array}{l}\text { Signal strength between } \\
\text { target node and anchor node }\end{array}$} & \multicolumn{4}{|c|}{$\begin{array}{l}\text { Real distance between } \\
\text { target node and anchor node }\end{array}$} \\
\hline $\mathbf{X}$ & $\mathbf{Y}$ & $\mathbf{A}$ & B & C & D & $\mathbf{A}$ & B & C & D \\
\hline 4 & 3 & 123.00 & 117.67 & 101.33 & 97.33 & 5.00 & 6.71 & 9.22 & 8.06 \\
\hline 3.5 & 3 & 125.33 & 79.00 & 99.33 & 99.33 & 4.61 & 7.16 & 9.55 & 7.83 \\
\hline 3 & 3 & 118.33 & 95.67 & 82.00 & 94.33 & 4.24 & 7.62 & 9.90 & 7.62 \\
\hline 3.5 & 3.5 & 101.67 & 101.00 & 88.33 & 97.00 & 4.95 & 7.38 & 9.19 & 7.38 \\
\hline 4 & 3.5 & 120.00 & 110.33 & 92.00 & 108.67 & 5.32 & 6.95 & 8.85 & 7.63 \\
\hline 4.5 & 3.5 & 112.00 & 100.00 & 104.33 & 100.67 & 5.70 & 6.52 & 8.51 & 7.91 \\
\hline 5 & 4 & 110.00 & 101.00 & 92.67 & 91.00 & 6.40 & 6.40 & 7.81 & 7.81 \\
\hline 4.5 & 4 & 92.00 & 108.00 & 108.67 & 108.00 & 6.02 & 6.80 & 8.14 & 7.50 \\
\hline 4 & 4 & 105.00 & 108.00 & 96.00 & 101.00 & 5.66 & 7.21 & 8.49 & 7.21 \\
\hline 4.5 & 4.5 & 110.00 & 101.67 & 83.00 & 105.00 & 6.36 & 7.11 & 7.78 & 7.11 \\
\hline 5 & 4.5 & 94.00 & 96.67 & 95.33 & 104.33 & 6.73 & 6.73 & 7.43 & 7.43 \\
\hline 5 & 5 & 101.00 & 80.33 & 92.67 & 107.00 & 7.07 & 7.07 & 7.07 & 7.07 \\
\hline 9.5 & 9.5 & 88.00 & 98.00 & 136.00 & 109.00 & 13.44 & 9.51 & 0.71 & 9.51 \\
\hline 9 & 9.5 & 78.00 & 92.33 & 186.00 & 90.00 & 13.09 & 9.55 & 1.12 & 9.01 \\
\hline 8.5 & 9.5 & 74.33 & 94.67 & 174.00 & 102.00 & 12.75 & 9.62 & 1.58 & 8.51 \\
\hline 8 & 9.5 & 76.67 & 99.67 & 161.33 & 103.00 & 12.42 & 9.71 & 2.06 & 8.02 \\
\hline 7.5 & 9.5 & 82.00 & 102.33 & 150.00 & 111.33 & 12.10 & 9.82 & 2.55 & 7.52 \\
\hline 7 & 9.5 & 71.00 & 60.67 & 150.00 & 102.00 & 11.80 & 9.96 & 3.04 & 7.02 \\
\hline 6.5 & 9.5 & 83.33 & 84.33 & 130.00 & 113.00 & 11.51 & 10.12 & 3.54 & 6.52 \\
\hline 6 & 9.5 & 95.00 & 84.00 & 121.67 & 113.67 & 11.24 & 10.31 & 4.03 & 6.02 \\
\hline 5.5 & 9.5 & 76.00 & 71.00 & 126.67 & 120.67 & 10.98 & 10.51 & 4.53 & 5.52 \\
\hline 5 & 9.5 & 61.67 & 84.67 & 110.00 & 126.00 & 10.74 & 10.74 & 5.02 & 5.02 \\
\hline 5 & 9 & 92.67 & 83.00 & 109.67 & 123.67 & 10.30 & 10.30 & 5.10 & 5.10 \\
\hline 5.5 & 9 & 80.33 & 69.00 & 120.67 & 121.00 & 10.55 & 10.06 & 4.61 & 5.59 \\
\hline 6 & 9 & 91.67 & 71.00 & 126.00 & 93.67 & 10.82 & 9.85 & 4.12 & 6.08 \\
\hline 7.5 & 9 & 84.33 & 73.33 & 144.33 & 106.67 & 11.72 & 9.34 & 2.69 & 7.57 \\
\hline 8 & 9 & 81.67 & 74.00 & 158.33 & 90.00 & 12.04 & 9.22 & 2.24 & 8.06 \\
\hline 8.5 & 9 & 62.33 & 97.00 & 165.00 & 109.00 & 12.38 & 9.12 & 1.80 & 8.56 \\
\hline 9 & 9 & 78.33 & 113.33 & 180.00 & 90.00 & 12.73 & 9.06 & 1.41 & 9.06 \\
\hline 8.5 & 8.5 & 88.00 & 91.00 & 162.00 & 97.67 & 12.02 & 8.63 & 2.12 & 8.63 \\
\hline 8 & 8.5 & 74.67 & 73.33 & 149.67 & 96.67 & 11.67 & 8.73 & 2.50 & 8.14 \\
\hline 7.5 & 8.5 & 95.33 & 113.67 & 143.00 & 88.33 & 11.34 & 8.86 & 2.92 & 7.65 \\
\hline 7 & 8.5 & 85.00 & 97.67 & 138.00 & 107.00 & 11.01 & 9.01 & 3.35 & 7.16 \\
\hline 6.5 & 8.5 & 88.00 & 82.00 & 131.67 & 114.00 & 10.70 & 9.19 & 3.81 & 6.67 \\
\hline 6 & 8.5 & 44.67 & 85.33 & 112.33 & 114.00 & 10.40 & 9.39 & 4.27 & 6.18 \\
\hline 5.5 & 8.5 & 80.00 & 96.00 & 117.33 & 114.67 & 10.12 & 9.62 & 4.74 & 5.70 \\
\hline 5 & 8.5 & 86.67 & 73.33 & 109.00 & 125.67 & 9.86 & 9.86 & 5.22 & 5.22 \\
\hline 5 & 8 & 101.00 & 64.00 & 95.00 & 126.00 & 9.43 & 9.43 & 5.39 & 5.39 \\
\hline 5.5 & 8 & 83.00 & 75.33 & 114.00 & 119.67 & 9.71 & 9.18 & 4.92 & 5.85 \\
\hline 6 & 8 & 71.67 & 86.67 & 119.33 & 114.00 & 10.00 & 8.94 & 4.47 & 6.32 \\
\hline 6.5 & 8 & 71.00 & 96.67 & 128.67 & 113.00 & 10.31 & 8.73 & 4.03 & 6.80 \\
\hline 7 & 8 & 85.00 & 55.00 & 138.00 & 90.00 & 10.63 & 8.54 & 3.61 & 7.28 \\
\hline 7.5 & 8 & 75.67 & 93.67 & 134.33 & 76.00 & 10.97 & 8.38 & 3.20 & 7.76 \\
\hline 7.5 & 7.5 & 88.67 & 100.67 & 136.33 & 95.00 & 10.61 & 7.91 & 3.54 & 7.91 \\
\hline
\end{tabular}




\begin{tabular}{|c|c|c|c|c|c|c|c|c|c|}
\hline \multicolumn{2}{|c|}{ Coordinate } & \multicolumn{4}{|c|}{$\begin{array}{l}\text { Signal strength between } \\
\text { target node and anchor node }\end{array}$} & \multicolumn{4}{|c|}{$\begin{array}{l}\text { Real distance between } \\
\text { target node and anchor node }\end{array}$} \\
\hline$x$ & $\mathbf{Y}$ & $\mathbf{A}$ & B & C & D & $\mathbf{A}$ & B & C & D \\
\hline 7 & 7.5 & 86.33 & 89.33 & 138.00 & 104.67 & 10.26 & 8.08 & 3.91 & 7.43 \\
\hline 6.5 & 7.5 & 82.33 & 41.67 & 124.00 & 111.33 & 9.92 & 8.28 & 4.30 & 6.96 \\
\hline 6 & 7.5 & 91.00 & 62.33 & 124.00 & 105.33 & 9.60 & 8.50 & 4.72 & 6.50 \\
\hline 5.5 & 7.5 & 95.00 & 89.33 & 113.00 & 119.00 & 9.30 & 8.75 & 5.15 & 6.04 \\
\hline 5 & 7.5 & 89.33 & 106.00 & 103.33 & 116.33 & 9.01 & 9.01 & 5.59 & 5.59 \\
\hline 5 & 7 & 92.00 & 52.00 & 104.00 & 119.67 & 8.60 & 8.60 & 5.83 & 5.83 \\
\hline 5.5 & 7 & 97.00 & 86.33 & 106.00 & 109.00 & 8.90 & 8.32 & 5.41 & 6.26 \\
\hline 6 & 7 & 60.67 & 101.33 & 125.33 & 108.00 & 9.22 & 8.06 & 5.00 & 6.71 \\
\hline 6.5 & 7 & 101.67 & 102.33 & 115.67 & 101.00 & 9.55 & 7.83 & 4.61 & 7.16 \\
\hline 7 & 7 & 95.33 & 92.00 & 124.67 & 99.00 & 9.90 & 7.62 & 4.24 & 7.62 \\
\hline 6.5 & 6.5 & 90.00 & 111.67 & 121.00 & 107.33 & 9.19 & 7.38 & 4.95 & 7.38 \\
\hline 5 & 6.5 & 101.00 & 51.00 & 96.33 & 122.00 & 8.20 & 8.20 & 6.10 & 6.10 \\
\hline 5 & 6 & 106.33 & 108.00 & 110.00 & 106.33 & 7.81 & 7.81 & 6.40 & 6.40 \\
\hline 5.5 & 6 & 102.67 & 73.67 & 102.67 & 101.67 & 8.14 & 7.50 & 6.02 & 6.80 \\
\hline
\end{tabular}

Journal of Industrial Engineering and Management, 2015 (www.jiem.org)

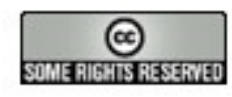

Article's contents are provided on a Attribution-Non Commercial 3.0 Creative commons license. Readers are allowed to copy, distribute and communicate article's contents, provided the author's and Journal of Industrial Engineering and Management's names are included.

It must not be used for commercial purposes. To see the complete license contents, please visit

http://creativecommons.org/licenses/by-nc/3.0/. 